\section{A Unique Case of Type-1 Facioscapulohumeral Muscular Dystrophy and Sarcomeric Hypertrophic Cardiomyopathy}

\section{Un caso único de distrofia muscular facioscapulohumeral tipo} 1 y miocardiopatía hipertrófica sarcomérica

\section{To the Editor,}

This report describes a unique case of genetically confirmed overlap of type-1 facioscapulohumeral muscular dystrophy (FSHD1) and obstructive sarcomeric hypertrophic cardiomyopathy (sHCM).

We present the case of a 37-year-old woman, diagnosed with FSHD1 and sHCM with substantial left ventricular (LV) hypertro- phy and severe symptomatic LV outflow tract obstruction. Molecular analysis confirmed the presence of contraction of the specific $18 \mathrm{~Kb}$ fragment in the $D 4 Z 4$ locus of chromosome $4 \mathrm{q}$ and the haplotype $4 \mathrm{qA}$, confirming the diagnosis of FSHD1, and the presence of the previously described c.1800.delA (p.Lys600Asnfs*) pathogenic mutation in the MYBPC3 gene, confirming the diagnosis of sHCM. ${ }^{1}$ The patient's father had the FSHD1 phenotype, although molecular analysis was not available. The patient's mother had the same pathogenic mutation on the MYBPC3 gene, although she had only a mild phenotype.

The first symptoms of FSHD1 arose at age 5 years with decreased strength of facial muscles and inability to smile or whistle. In the third decade of life, an asymmetric reduction in upper limb strength developed. Neurological evaluation was remarkable for typical FSHD1 phenotype with bilateral peripheral 
facial paresis, atrophy of the pectoral muscles and winged scapulae. The electromyogram showed myopathic changes in the orbicularis and trapezius muscles. Creatinine kinase levels were normal.

Also in the third decade of life, the patient developed effort dyspnea (New York Heart Association III functional class) and presyncope. $\mathrm{N}$-terminal pro-B-type natriuretic peptide levels were increased $(400 \mathrm{pg} / \mathrm{mL})$. Electrocardiography revealed LV hypertrophy (voltage criteria) and T-wave inversion in the inferior leads. Serial echocardiograms showed septal asymmetric hypertrophy (interventricular septum $=25 \mathrm{~mm}$, pulse wave $=13 \mathrm{~mm}$ ), severe LV outflow tract obstruction (rest peak gradient $=40 \mathrm{mmHg}$; exercise stress peak gradient $=100 \mathrm{mmHg}$ ), anterior systolic motion of the anterior mitral leaflet associated with moderate mitral regurgitation, and grade II diastolic dysfunction. Episodes of nonsustained monomorphic ventricular tachycardia were found on 24-hour Holter monitoring. A single chamber implantable cardioverter-defibrillator was implanted.

Titration of beta-blocker therapy was not tolerated (hypotension) and alcohol septal ablation was considered. Coronary angiography was performed, showing the presence of 2 septal arteries that were candidates for alcohol septal ablation. Although the first septal artery irrigated the region of interest, the contrast opacification was suboptimal. The second septal artery irrigated the right portion of the septum, inferior wall, and posteromedial papillary muscle. We considered that the risk-benefit ratio was unfavorable and the patient underwent surgical myectomy. The postoperative period was complicated by complete atrioventricular block, and the monochamber implantable cardioverter-defibrillator was upgraded to a dual-chamber implantable cardioverterdefibrillator.

At 2 years of follow-up, the patient is only mildly symptomatic (New York Heart Association functional class I-II), with no dysrhythmic events. An echocardiogram showed a decrease in the asymmetric septal hypertrophy (interventricular septum $=14 \mathrm{~mm}$, pulse wave $=13 \mathrm{~mm}$ ) and a resting peak gradient of $40 \mathrm{mmHg}$, but with no further increase on exercise stress testing.

Both FSHD1 due to contraction of the D4Z4 locus in the subtelomeric $4 \mathrm{q} 35$ region and $\mathrm{SHCM}$ due to $M Y B P C 3$ pathogenic mutation c.1800.delA (p.Lys600Asnfs*) were identified in the patient described. To the best of our knowledge, there are no published cases describing the co-occurrence of FSHD1 and sHCM. Epidemiologic studies suggest that the frequency of FSHD is about $1: 20000$, while the frequency of sHCM is estimated to be $1: 500 .^{2}$ Thus, the chance of being affected by both disorders is about 1:10 000000 .

In contrast to other muscular dystrophies such as Duchenne's where the development of dilated cardiomyopathy and heart failure are common, cardiac involvement in FSHD1 is rare and is characterized by conduction abnormalities and supraventricular dysrhythmias. Three cases in the medical literature describe cardiomyopathy associated with FSHD1. ${ }^{3-5}$ Finsterer et al. ${ }^{3}$ describe a 50-year-old FSHD1 and hypertensive patient (blood pressure $150 / 90 \mathrm{mmHg}$ ) with electrocardiographic abnormalities and left ventricular hypertrophy. Emmrich et al. ${ }^{4}$ report a 71 -yearold woman with FSHD1 and progressive heart failure whose autopsy revealed normal muscle mass volume and histological changes seen in primary cardiomyopathies. In both cases, no molecular testing was performed and conflicting clinical data exist about the presence of cardiomyopathy. The last case, by Tsuji et al., ${ }^{5}$ concerns a 38-year-old FSHD1 patient with associated retinal vasculopathy and neurosensorial hearing impairment, whose echocardiogram showed mild dilatation of the LV associated with poor contractility; cardiac histopathology revealed marked disarray and mild fibrosis, suggesting a dilated phase of sHCM rather than dilated cardiomyopathy. Also, no molecular testing was reported.

In addition to the pathogenic mutation in the $M Y B P C 3$ gene, our patient had the following variants of unknown significance: p.Cys5939Tyr and p.Lys14140Val in the TTN gene, and p.Tyr904Cys in the Ryr2 gene. Her mother also had the same variants of unknown significance in the TTN gene. Because of the genetic complexity of sHCM, clinical interpretation of a large number of rare variants of unknown significance with uncertain effects on gene function identified with current sequencing technology testing is a real challenge. Ideally, novel variants of unknown significance should undergo functional studies, but these are timeconsuming, costly, and often impractical in the clinical setting. Similarly, cosegregation analysis within families may be helpful, but is uninformative in small pedigrees and is often difficult to orchestrate. $^{6}$

This case report describes a unique patient with genetically confirmed overlapping diagnoses of FSHD1 and severe obstructive sHCM. Proper clinical and genetic evaluation of these overlapping patients is critical for their management. This case also highlights the importance of cardiological evaluation in patients with muscular dystrophies.

Gustavo Lima da Silva,* Tatiana Guimarães, Fausto J. Pinto, and Dulce Brito

Cardiology Department, Santa Maria University Hospital, Lisbon Academic Medical Center, Cardiovascular Center, University of Lisbon, Lisbon, Portugal

* Corresponding author:

E-mail address: gustavolssilva@gmail.com (G. Lima da Silva).

Available online 8 July 2017

\section{REFERENCES}

1. Brito D, Miltenberger-Miltenyib G, Vale Pereira S, Silva D, Nunes Diogo A, Madeira H. Sarcomeric hypertrophic cardiomyopathy: Genetic profile in a Portuguese population. Rev Port Cardiol. 2012;31:577-587.

2. Maron BJ, Gardin JM, Flack JM, Gidding SS, Kurosaki TT, Bild DE. Prevalence of hypertrophic cardiomyopathy in a general population of young adults. Circulation. 1995;92:785-789.

3. Finsterer J, Stöllberger C, Meng G. Cardiac involvement in facioscapulohumeral muscular dystrophy. Cardiology. 2005;103:81-83.

4. Emmrich P, Ogunlade V, Gradistanac T, Daneschnejad S, Koch MC, Schober R. Facioscapulohumeral muscle dystrophy and heart disease. Z Kardiol. 2005;94: 348-354.

5. Tsuji M, Kinoshita M, Imai Y, Kawamoto M, Kohara N. Facioscapulohumeral muscular dystrophy presenting with hypertrophic cardiomyopathy: a case study. Neuromuscul Disord. 2009;19:140-142.

6. Lopes LR, Zekavati A, Syrris P, et al. Genetic complexity in hypertrophic cardiomyopathy revealed by high-throughput sequencing. J Med Genet. 2013;50: 228-239.

http://dx.doi.org/10.1016/j.rec.2017.06.007

1885-5857|

(c) 2017 Sociedad Española de Cardiología. Published by Elsevier España, S.L.U. All rights reserved. 